\title{
INVARIANCE PROPERTIES OF SCHOENBERG'S TONE ROW SYSTEM
}

\author{
JAMES A. FILL and ALAN J. IZENMAN
}

(Received 13 July 1978)

(Revised 25 October 1978)

\begin{abstract}
This paper organizes in a systematic manner the major features of a general theory of $m$-tone rows. A special case of this development is the twelve-tone row system of musical composition as introduced by Arnold Schoenberg and his Viennese school. The theory as outlined here applies to tone rows of arbitrary length, and can be applied to microtonal composition for electronic media.
\end{abstract}

\section{Introduction}

Musical composition in the twentieth century has been enlivened by Arnold Schoenberg's introduction of a structured system which emphasizes its serial and atonal nature. Schoenberg called his system "A method of composing with twelve tones which are related only with one another" [12] page 107. Although Schoenberg himself regarded his work as the logical outgrowth of tendencies inherent in the development of Austro-German music during the previous one hundred years, it has been criticized as purely "abstract and mathematical cerebration" and a certain amount of controversy still surrounds the method.

The fundamental building-block in Schoenberg's system is the twelve-tone row, a specific linear ordering of all twelve notes-C, $C^{\sharp}, D, E^{b}, E, F, F^{*}, G, G^{\#}, A$, $\mathrm{B}^{b}$, and $\mathrm{B}-$ of the equally tempered chromatic scale, each note appearing once and only once within the row. A work, or section of a work, based on a particular twelve-tone row (usually designated as the original or prime) is permitted to include all possible transpositions of the prime, together with those of its retrograde, its inversion and its retrograde-inversion. (See the next section for explanations of this terminology.) Furthermore, each note can be stated in any register. 
Theoretically, no note is more important than any other note, and none of the four forms has primacy over the other three.

Although certain modifications have been made to this set of rules in recent years, the basic principles have been followed fairly faithfully. Notable composers who have used the twelve-tone row system in their musical compositions include Alban Berg, Anton Webern, Karlheinz Stockhausen, Pierre Boulez, Igor Stravinsky and Milton Babbitt. A more detailed description and a critical examination of the method may be found in Perle [11].

The aim of this paper is to present the algebraic flavour and structure of Schoenberg's system of musical composition. Numerous studies have appeared in the musical literature dealing with various music-analytic properties of twelvetone rows, most notably those of Milton Babbitt ([1], [2], [3], [4] and [5]), who has given the subject a great deal of its nomenclature. However, none of these studies has attempted to determine the number of twelve-tone rows which possess certain interesting properties, such as being invariant under any of the operations of transposition, retrograde, inversion or retrograde-inversion. The interrelationships between the four forms of the prime twelve-tone row can be studied through the set-complex, an ingenious tool devised by Babbitt in [1]. Each twelvetone row generates its own set-complex which contains all of its various forms; it is of interest, therefore, to determine how many distinct set-complexes are generated by the complete set of all 12 ! twelve-tone rows.

Such questions are of considerable importance to the musician who wishes to compose in the twelve-tone row genre. In order to answer these questions, we analyse the twelve-tone row system within the more general framework of $m$-tone rows, where $m$ is an arbitrary integer. The elements of such an $m$-tone row are some permutation of the notes of an $m$-tone scale. The advantages of such a generalization are not only mathematical; with the advent of electronic music produced by a suitably programmed computer or synthesizer, the study and composition of microtonal works in which intervals between notes are smaller than a semi-tone is now feasible.

A list of all twelve-tone compositions by Schoenberg together with their associated twelve-tone rows may be found in [10].

\section{Notation}

For notational simplicity and technical ease, we shall substitute the integers 0 through $m-1$ for the ordered succession of $m$ ascending notes that constitute the $m$-note scale in question. Such a representation is standard for twelve-tone row theory (where $m=12$; see Chapter 1 of Perle [11]) and is probably necessary for the theory of microtonal composition. 
Let $m>2$ be an integer and let

$$
\Pi=\left(\begin{array}{ccccc}
0 & 1 & 2 & \ldots & m-1 \\
\pi_{0} & \pi_{1} & \pi_{2} & \ldots & \pi_{m-1}
\end{array}\right)
$$

be a permutation of $Z_{m}=\{0,1, \ldots, m-1\}$. Then $\Pi$ is called an m-tone row (or $m$-phonic sequence). The set of all $m ! m$-tone rows is denoted by $\mathscr{P}_{m}$. For example, the twelve-tone row

$$
\mathrm{II}=\left(\begin{array}{rrrrrrrrrrrr}
0 & 1 & 2 & 3 & 4 & 5 & 6 & 7 & 8 & 9 & 10 & 11 \\
9 & 10 & 3 & 11 & 4 & 6 & 0 & 1 & 7 & 8 & 2 & 5
\end{array}\right)
$$

which can also be written in the familiar cycle notation as

$$
\Pi=\left(\begin{array}{lllllllllll}
0 & 9 & 8 & 7 & 1 & 10 & 2 & 3 & 11 & 5 & 6
\end{array}\right)(4),
$$

appears in Schoenberg's Violin Concerto of 1936.

We now define three operations on $\Pi$. Let $\alpha$ be the cyclic permutation $\left(\begin{array}{llll}0 & 1 & \ldots & m-1\end{array}\right)$, which corresponds to a raising each tone by one unit. Then the transposition of $\Pi$ by the integer $a$ is the $m$-tone row $\Pi \alpha^{a}$. Thus the transposition of $\Pi$ in $\left(^{*}\right)$ above by 3 yields the twelve-tone row

$$
\begin{aligned}
& \Pi \alpha^{3}=\left(\begin{array}{rrrrrrrrrrrr}
0 & 1 & 2 & 3 & 4 & 5 & 6 & 7 & 8 & 9 & 10 & 11 \\
0 & 1 & 6 & 2 & 7 & 9 & 3 & 4 & 10 & 11 & 5 & 8
\end{array}\right) \\
& =(0)(1)(2 \quad 6 \quad 3)(4 \quad 7)(5 \quad 9 \quad 11 \quad 8 \quad 10) \text {. }
\end{aligned}
$$

Next, let $\beta$ be the permutation $(0 m-1)(1 m-2)(2 m-3) \ldots$, which corresponds to reversing the order of the elements. Then the retrograde (or crab) of $\Pi$ is the $m$-tone row $\beta \Pi$. Thus the retrograde of $\Pi$ in $\left(^{*}\right)$ above is

$$
\begin{aligned}
& \beta \Pi=\left(\begin{array}{rrrrrrrrrrrr}
0 & 1 & 2 & 3 & 4 & 5 & 6 & 7 & 8 & 9 & 10 & 11 \\
5 & 2 & 8 & 7 & 1 & 0 & 6 & 4 & 11 & 3 & 10 & 9
\end{array}\right)
\end{aligned}
$$

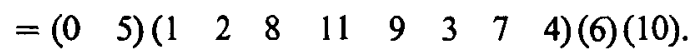

Note that $\beta^{2}=1$. Finally, let $\gamma$ be the permutation $(1 m-1)(2 m-2)(3 m-3) \ldots$ Then the negative of $\Pi$ is the $m$-tone row $\Pi \gamma$. The negative of $\Pi$ in $\left(^{*}\right)$ above is

$$
\begin{aligned}
\Pi \gamma & =\left(\begin{array}{rrrrrrrrrrrr}
0 & 1 & 2 & 3 & 4 & 5 & 6 & 7 & 8 & 9 & 10 & 11 \\
3 & 2 & 9 & 1 & 8 & 6 & 0 & 11 & 5 & 4 & 10 & 7
\end{array}\right) \\
& =\left(\begin{array}{lllllllll}
0 & 3 & 1 & 2 & 9 & 4 & 8 & 5 & 6
\end{array}\right)\left(\begin{array}{lll}
7 & 11
\end{array}\right)(10) .
\end{aligned}
$$


Note that $\alpha \gamma=\gamma \alpha^{-1}$. The inversion of an $m$-tone row $\Pi$ is now the $m$-tone row $\Pi \alpha^{-2 \pi_{0}} \gamma\left(=\Pi \gamma \alpha^{2 \pi_{0}}\right)$. For the $\Pi$ in $\left(^{*}\right)$ above, the inversion is

$$
\begin{aligned}
\Pi \alpha^{6} \gamma & =\left(\begin{array}{rrrrrrrrrrrr}
0 & 1 & 2 & 3 & 4 & 5 & 6 & 7 & 8 & 9 & 10 & 11 \\
9 & 8 & 3 & 7 & 2 & 0 & 6 & 5 & 11 & 10 & 4 & 1
\end{array}\right) \\
& =\left(\begin{array}{llllllll}
0 & 9 & 10 & 4 & 2 & 3 & 7 & 5
\end{array}\right)\left(\begin{array}{llll}
1 & 8 & 11
\end{array}\right)(6) .
\end{aligned}
$$

It is worth noting that this last definition is not the usual way in which inversion is defined. Perle [11] page 3, for example, calls the negative of a tone row $\Pi$ the inversion of $\Pi$. However, for certain reasons which will become apparent later, we wish to set the first element of the inversion of $\Pi$ equal to the first element of $\Pi$ itself. This requirement leads naturally to the above alternative definition. The retrograde-inversion (or crab-inversion) of $\Pi$ is the retrograde of the inversion of $\Pi$ and is the $m$-tone row $\beta \Pi \alpha^{-2 \pi_{0}} \gamma$. Thus the retrograde-inversion of $\Pi$ in (*) above is

$$
\begin{aligned}
\beta \Pi \alpha^{6} \gamma & =\left(\begin{array}{rrrrrrrrrrrr}
0 & 1 & 2 & 3 & 4 & 5 & 6 & 7 & 8 & 9 & 10 & 11 \\
1 & 4 & 10 & 11 & 5 & 6 & 0 & 2 & 7 & 3 & 8 & 9
\end{array}\right) \\
& =\left(\begin{array}{llllllllll}
0 & 1 & 4 & 5 & 6
\end{array}\right)\left(\begin{array}{llllll}
2 & 10 & 8 & 7
\end{array}\right)\left(\begin{array}{llll}
3 & 11 & 9
\end{array}\right) .
\end{aligned}
$$

It will be convenient to use the following abbreviated version of an $m$-tone row $\Pi$ in the discussion below. Instead of writing $\Pi$ in the form (1) above, we shall henceforth write either

$$
\Pi=\left(\pi_{0}, \pi_{1}, \pi_{2}, \ldots, \pi_{m-1}\right)
$$

or simply

$$
\begin{array}{lllll}
\pi_{0} & \pi_{1} & \pi_{2} & \ldots & \pi_{m-1}
\end{array}
$$

the permutational ordering of the elements being understood.

The following results are now straightforward consequences of the above definitions.

(i) The retrograde of a transposition is the same transposition of the retrograde; that is, $\beta\left(\Pi \alpha^{a}\right)=(\beta \Pi) \alpha^{a}$.

(ii) The inversion of a transposition is the same transposition of the inversion; that is, $\left(\Pi \alpha^{a}\right) \alpha^{-2\left(\pi_{0}+a\right)} \gamma=\left(\Pi \alpha^{-2 \pi_{0}} \gamma\right) \alpha^{a}$.

(iii) The retrograde-inversion of a transposition is the same transposition of the retrograde-inversion; that is, $\beta\left(\Pi \alpha^{a}\right) \alpha^{-2\left(\pi_{0}+a\right)} \gamma=\left(\beta \Pi \alpha^{-2 \pi_{0}} \gamma\right) \alpha^{a}$. 
Furthermore, because of the way in which we have defined the inversion of $\Pi$, the retrograde of the inversion of $\Pi$ is not in general the same as the inversion of the retrograde of $\Pi$; that is,

$$
\beta\left(\Pi \alpha^{-2 \pi_{0}} \gamma\right)=\beta \Pi \gamma \alpha^{2 \pi_{0}} \neq \beta \Pi \gamma \alpha^{2 \pi_{m-1}}=(\beta \Pi) \alpha^{-2 \pi_{m-1}} \gamma
$$

unless $m$ is even and. $\left|\pi_{m-1}-\pi_{0}\right|=m / 2$. On the other hand, however, the usual definition of the inversion of $\Pi$, namely $\Pi \gamma$, always results in their equality.

\section{The set-complex}

The four forms of an $m$-tone row and the $m$ transpositions of each form can be related to each other in the following useful way. The set-complex, $\square(\Pi)$, corresponding to a particular $m$-tone row $\Pi$ is the set of all $m$-tone rows of the four forms $\Pi \alpha^{a}, \beta \Pi \alpha^{a}, \Pi \alpha^{-2 \pi_{0}} \gamma \alpha^{a}$ and $\beta \Pi \alpha^{-2 \pi_{0}} \gamma \alpha^{a}$, and may be represented as an $m \times m$ square (hence the symbol $\square(\Pi)$ ) whose entries are arranged in a "checkerboard" fashion as follows. Along the top line write the original $m$-tone row $\Pi$. Next, write the $m$-tone row corresponding to the inversion of $\Pi$, namely $I(\Pi)=\Pi \alpha^{-2 \pi_{0}} \gamma$, down the first column; from the way we have defined the inversion of $\Pi$, the starting element of both $\Pi$ and its inversion will be the same. The square is completed by writing in each line the transposition of $\Pi$ which starts with the element of the inversion in the first column. As an example we give in Table 1 the set-complex of Schoenberg's Fourth String Quartet for which $m=12$. The rows reading from left to right give all 12 transpositions of $\Pi$; the columns reading from top to bottom give all 12 transpositions of $I(\Pi)=\Pi \alpha^{-2 \pi_{0}} \gamma$; the rows reading from right to left give all 12 transpositions of $R(\Pi)=\beta \Pi$; and the columns reading from bottom to top give all 12 transpositions of $R I(\Pi)=\beta \Pi \alpha^{-2 \pi_{0}} \gamma$.

TABLE 1

\begin{tabular}{l|rrrrrrrrrrrr}
\hline & 0 & 1 & 2 & 3 & 4 & 5 & 6 & 7 & 8 & 9 & 10 & 11 \\
\hline$\Pi$ & 2 & 1 & 9 & 10 & 5 & 3 & 4 & 0 & 8 & 7 & 6 & 11 \\
$\Pi \alpha$ & 3 & 2 & 10 & 11 & 6 & 4 & 5 & 1 & 9 & 8 & 7 & 0 \\
$\Pi \alpha^{5}$ & 7 & 6 & 2 & 3 & 10 & 8 & 9 & 5 & 1 & 0 & 11 & 4 \\
$\Pi \alpha^{4}$ & 6 & 5 & 1 & 2 & 9 & 7 & 8 & 4 & 0 & 11 & 10 & 3 \\
$\Pi \alpha^{9}$ & 11 & 10 & 6 & 7 & 2 & 0 & 1 & 9 & 5 & 4 & 3 & 8 \\
$\Pi \alpha^{11}$ & 1 & 0 & 8 & 9 & 4 & 2 & 3 & 11 & 7 & 6 & 5 & 10 \\
$\Pi \alpha^{10}$ & 0 & 11 & 7 & 8 & 3 & 1 & 2 & 10 & 6 & 5 & 4 & 9 \\
$\Pi \alpha^{2}$ & 4 & 3 & 11 & 0 & 7 & 5 & 6 & 2 & 10 & 9 & 8 & 1 \\
$\Pi \alpha^{6}$ & 8 & 7 & 3 & 4 & 11 & 9 & 10 & 6 & 2 & 1 & 0 & 5 \\
$\Pi \alpha^{7}$ & 9 & 8 & 4 & 5 & 0 & 10 & 11 & 7 & 3 & 2 & 1 & 6 \\
$\Pi \alpha^{8}$ & 10 & 9 & 5 & 6 & 1 & 11 & 0 & 8 & 4 & 3 & 2 & 7 \\
$\Pi \alpha^{3}$ & 5 & 4 & 0 & 1 & 8 & 6 & 7 & 3 & 11 & 10 & 9 & 2 \\
& & & & & & & & & & & &
\end{tabular}


Notice that the elements of such a "checkerboard" representation form a Latin square (see [6]). However, this square array is just a convenient method of writing down all of the $4 m$ possible $m$-tone rows that can be generated from a given $m$-tone row, and is not of any special interest by itself. The fact that $\square(\Pi)$ is a Latin square is not used in the remaining sections of this paper.

Duplication of $m$-tone rows within a given set-complex will now occur if any of the following relationships hold for some $a$ :

$$
\begin{aligned}
\Pi & =\Pi \alpha^{a} \\
\Pi & =\beta \Pi \alpha^{a} \\
\Pi & =\Pi \alpha^{-2 \pi_{0}} \gamma \alpha^{a} \\
\Pi & =\beta \Pi \alpha^{-2 \pi_{0}} \gamma \alpha^{a} \\
\beta \Pi & =\Pi \alpha^{-2 \pi_{0}} \gamma \alpha^{a} \\
\beta \Pi & =\beta \Pi \alpha^{-2 \pi_{0}} \gamma \alpha^{a} \\
\Pi \alpha^{-2 \pi_{0}} \gamma & =\beta \Pi \alpha^{-2 \pi_{0}} \gamma \alpha^{a} .
\end{aligned}
$$

These relationships express the possible invariance properties of an $m$-tone row under the operations of transposition, retrograde, inversion, retrograde-inversion, and of all combinations of such operations. The question is now which of these invariances can occur and which cannot, and of those that can, to characterize them and determine their exact number.

Clearly, an $m$-tone row can never be a nonzero transposition of itself; that is, $\Pi \neq \Pi \alpha^{a}$ if $a \bmod m \neq 0$. Moreover, an $m$-tone row can never be a transposition of its own inversion; that is, for all $a, \Pi \neq \Pi \alpha^{-2 \pi_{0}} \gamma \alpha^{a}$. For, if

$$
\Pi=\Pi \alpha^{-2 \pi_{0}} \gamma \alpha^{a}=\Pi \gamma \alpha^{2 \pi_{0}+a},
$$

then $\gamma=\alpha^{-2 \pi_{0}-a}$, which is a contradiction. Relation (6) can also never occur since (3) can never occur. This eliminates the possibilities of (1), (3) and (6) above.

On the other hand, if an $m$-tone row $\Pi$ is a nonzero transposition of its own retrograde (that is (2) holds), then $m$ is even and $\Pi$ is the transposition of that retrograde by $m / 2$. For, suppose that $\Pi=\beta \Pi \alpha^{a}$. Then $\beta \Pi=\Pi \alpha^{a}$, whence $\Pi=\Pi \alpha^{2 a}$ and $2 a \bmod m=0$. This last implies that $m$ is even and that $a \bmod m=m / 2$, as asserted. With regard to (4) above, an $m$-tone row will be a transposition of its own retrograde-inversion if and only if $\Pi=\beta \Pi \gamma \alpha^{c}$ for some $c \in Z_{m}$; furthermore, if $m$ is even, then $c$ will be odd. To see this, note that

$$
\sum_{i=0}^{m-1} \pi_{i}=m(m-1) / 2 \equiv m / 2 \quad(\bmod m)
$$


If $\Pi=\beta \Pi \gamma \alpha^{c}$ holds, then $\pi_{i} \equiv c-\pi_{m-1-i}(\bmod m), 0 \leqslant i \leqslant(m / 2)-1$, and so

$$
\sum_{i=0}^{m-1} \pi_{i} \equiv m c / 2 \quad(\bmod m) .
$$

Thus $c$ is odd as asserted. Relation (5) follows directly from (4), while (7) (with $a$ replaced by $-a$ ) follows from (2). Finally, statements (2) (that $\Pi$ is a transposition of its own retrograde) and (4) (that $\Pi$ is a transposition of its own retrograde-inversion) are mutually-exclusive by virtue of the fact that (3) can never occur.

From the above results, we make the following observations. The set-complex $\square(\Pi)$ corresponding to a particular $m$-tone row $\Pi$ by definition contains $4 m$ $m$-tone rows. The above results suggest, however, that for certain rows $\Pi$, the number of distinct $m$-tone rows contained in $\square(\Pi)$ may be smaller than $4 m$. Indeed, we have seen that duplications will occur in $\square(\Pi)$ if and only if the $m$-tone row $\Pi$ satisfies either of the following two conditions:

$$
\begin{aligned}
& m \text { is even and } \Pi=\beta \Pi \alpha^{m / 2}, \\
& \Pi=\beta \Pi \gamma \alpha^{c} \text {, for some } c \in Z_{m} ; \text { and if } m \text { is even, then } c \text { must be odd. }
\end{aligned}
$$

Sections 4 and 5 below are concerned with the determination of the exact number of $m$-tone rows for which conditions (1) or (2) hold. Moreover, the above remarks allow us to examine cases (1) and (2) separately.

\section{Results for $m$ even}

Let $m>2$ be an even integer throughout this section. We will sometimes write $2 k$ for $m$.

THEOREM 4.1. There are exactly $2^{k} k ! m$-tone rows $\Pi$ for which $\Pi=\beta \Pi \alpha^{k}$.

Proof. Now $\Pi=\beta \Pi \alpha^{k}$ if and only if $\Pi$ is of the form

$$
\Pi=\left(\pi_{0}, \pi_{1}, \ldots, \pi_{k-1},\left(\pi_{k-1}+k\right) \bmod m, \ldots,\left(\pi_{1}+k\right) \bmod m,\left(\pi_{0}+k\right) \bmod m\right) .
$$

All such sequences may be generated without repetition according to the following two-step process. For each $i=0,1, \ldots, k-1$ : (i) successively, for $j=0,1, \ldots, k-1$, choose the pair of positions $\pi_{i}$ and $\pi_{2 k-1-i}$ to be occupied by the pair of values $j$ and $j+k$; then (ii) for each $j=0,1, \ldots, k-1$, decide whether $\pi_{i}=j$ and $\pi_{2 k-1-i}=j+k$, or whether $\pi_{i}=j+k$ and $\pi_{2 k-1-i}=j$. There are $k$ ! possible results of step (i), and for each of those there are $2^{k}$ possible results of step (ii). 
THEOREM 4.2. There are exactly $k 2^{k} k ! m$-tone rows $\Pi$ for which there exists an odd constant $c \in Z_{m}$ such that $\Pi=\beta \Pi \gamma \alpha^{c}$.

Proof. We first prove that there are $2^{k} k ! m$-tone rows $\Pi$ for which $\Pi=\beta \Pi \gamma \alpha$. Now this latter equality holds if and only if $\Pi$ is of the form

$$
\Pi=\left(\pi_{0}, \pi_{1}, \ldots, \pi_{k-1},\left(1-\pi_{k-1}\right) \bmod m, \ldots,\left(1-\pi_{1}\right) \bmod m,\left(1-\pi_{0}\right) \bmod m\right) .
$$

All such sequences may be generated without repetition according to the following two-step process. For each $i=0,1, \ldots, k-1$, (i) successively, for $j=1,2, \ldots, k$, choose the pair of positions $\pi_{i}$ and $\pi_{2 k-1-i}$ to be occupied by the pair of values $j$ and $(1-j) \bmod m$; then (ii) for each $j=1,2, \ldots, k$, decide whether $\pi_{i}=j$ and $\pi_{2 k-1-i}=(1-j) \bmod m$, or whether $\pi_{i}=(1-j) \bmod m$ and $\pi_{2 k-1-i}=j$. There are $k$ ! possible results of step (i), and for each of those there are $2^{k}$ possible results of step (ii). To complete the proof, observe that $\Pi=\beta \Pi_{\gamma \alpha^{c}}$ holds if and only if $\Pi \alpha^{-d}=\beta \Pi \alpha^{-d} \gamma \alpha$, where $c=2 d+1$. The $k$ choices for $c$ (that is, for $d$ ) gives the factor of $k$ in $k 2^{k} k$ !.

An important example of a composition in which the prime twelve-tone row $I$ is a transposition of its own retrograde-inversion is that of Schoenberg's Suite, Opus 29, written in 1926. The twelve-tone row in question, transposed by 9 so that its first note is $\mathrm{C}$ (or 0 ), is

$$
\Pi=(0,1,4,5,8,9,2,3,6,7,10,11) ;
$$

in this case, $\Pi=\beta \Pi \gamma \alpha^{11}$. (Note also that for this example the sequence of 11 intervals between the 12 notes, namely $(1,3,1,3,1,5,1,3,1,3,1)$, is symmetric. Similar relationships between intervals and notes are explored in detail in [7].)

Contained in the above theorems is the following:

COROLLARY 4.3. There are exactly $2^{k}(k+1)$ ! m-tone rows $\Pi$ for which $\square(\Pi)$ has fewer than $4 m$ distinct members and, in such cases, the cardinality of $\square(\Pi)$ is $2 m$.

THEOREM 4.4. If $\square\left(\mathscr{P}_{m}\right)=\left\{\square(\Pi): \Pi \in \mathscr{P}_{m}\right\}$, then $\square\left(\mathscr{P}_{m}\right)$ has cardinality $\left(m !+2^{k}(k+1) !\right) /(4 m)$.

Proof. Let us say that two $m$-tone rows $\Pi$ and $\Pi^{\prime}$ are equivalent if $\square(\Pi)=\square\left(\Pi^{\prime}\right)$. The number of equivalence classes is then the cardinality of $\square\left(\mathscr{P}_{m}\right)$. Now $\Pi$ and $\Pi^{\prime}$ are equivalent if and only if (at least) one of the following four statements holds for some $a \in Z_{m}$ : (i) $\Pi^{\prime}=\Pi \alpha^{a}$; (ii) $\Pi^{\prime}=\beta \Pi \alpha^{a}$; (iii) $\Pi^{\prime}=\Pi \alpha^{-2 \pi_{0}} \gamma \alpha^{a}$; (iv) $\Pi^{\prime}=\beta \Pi \alpha^{-2 \pi_{0}} \gamma \alpha^{a}$. Let $G$ be the group of $4 m$ different permutations $\sigma_{i, a}$ 
with $1 \leqslant i \leqslant 4$ and $a \in Z_{m}$ given by

$$
\begin{aligned}
\sigma_{1, a}: & \Pi \rightarrow \Pi \alpha^{a}, \\
\sigma_{2, a}: & \Pi \rightarrow \beta \Pi \alpha^{a}, \\
\sigma_{3, a}: & \Pi \rightarrow \Pi \alpha^{-2 \pi_{0}} \gamma \alpha^{a}, \\
\sigma_{4, a}: & \Pi \rightarrow \beta \Pi \alpha^{-2 \pi_{0}} \gamma^{a} \alpha^{a} .
\end{aligned}
$$

The group operation here is composition of functions. It is easily verified that $G$ is indeed a permutation group, and that the equivalence relation induced by $G$ on $\mathscr{P}_{m}$ results in the equivalence of $\Pi$ and $\Pi^{\prime}$ if and only if $\square(I I)=\square\left(\Pi^{\prime}\right)$. According to Burnside's Lemma (see [9] page 136), the number of equivalence classes is

$$
\sum_{\sigma \in G} \psi(\sigma) /|G|
$$

where $|G|$ is the order of $G$ (here $|G|=4 m$ ) and $\psi(\sigma)$ is the number of elements of $\mathscr{P}_{m}$ which are invariant under $\sigma$. Clearly, $\psi\left(\sigma_{1,0}\right)=\left|\mathscr{P}_{m}\right|=m$ ! and $\psi\left(\sigma_{1, a}\right)=0$ if $a \neq 0$. Furthermore, $\psi\left(\sigma_{2, a}\right)=0$ unless $a=m / 2$, in which case

$$
\psi\left(\sigma_{2, a}\right)=\psi\left(\sigma_{2, m / 2}\right)=2^{k} k !
$$

by Theorem 4.1. Similarly, $\psi\left(\sigma_{3, a}\right)=0$ for all $a$. Finally, $\sum_{a=0}^{m-1} \psi\left(\sigma_{4, a}\right)=k 2^{k} k$ ! by Theorem 4.2. Thus, the number of equivalence classes is

$$
\left|\square\left(\mathscr{P}_{m}\right)\right|=\left(m !+2^{k} k !+k 2^{k} k !\right) /(4 m)=\left(m !+2^{k}(k+1) !\right) /(4 m) .
$$

THEOREM 4.5. The cardinality of $\left\{\square(\Pi): \Pi \in \mathscr{P}_{m}\right.$ and $\left.|\square(\Pi)|<4 m\right\}=\{\square(\Pi)$ : $\Pi \in \mathscr{P}_{m}$ and $\left.|\square(\Pi)|=2 m\right\}$ is $2^{k-1}(k+1) ! / m$.

Proof. Similar to that of Theorem 4.4.

\section{Results for $m$ odd}

Let $m>2$ be an odd integer throughout this section. We shall sometimes write $2 k+1$ for $m$.

THEOREM 5.1. There are precisely $m 2^{k} k ! m$-tone rows $\Pi$ for which there exists $a$ constant $c \in Z_{m}$ such that $\Pi=\beta \Pi \gamma \alpha^{c}$.

Proof. We first prove that there are $2^{k} k ! m$-tone rows $\Pi$ for which $\Pi=\beta \Pi \gamma$. Indeed, $\Pi=\beta \Pi \gamma$ if and only if $\Pi$ is of the form

$$
\Pi=\left(\pi_{0}, \pi_{1}, \ldots, \pi_{k-1}, 0,-\pi_{k-1} \bmod m, \ldots,-\pi_{1} \bmod m,-\pi_{0} \bmod m\right) .
$$


All such sequences may be generated without repetition according to the following two-step process. For each $i=0,1, \ldots, k-1$ : (i) successively, for $j=1,2, \ldots, k$, choose the pair of positions $\pi_{i}$ and $\pi_{m-1-i}$ to be occupied by the pair of values $j$ and $-j \bmod m$; then (ii) for each $j=1,2, \ldots, k$, decide whether $\pi_{i}=j$ and $\pi_{m-1-i}=-j \bmod m$, or whether $\pi_{i}=-j \bmod m$ and $\pi_{m-1-i}=j$. There are $k$ ! possible results of step (i), and for each of those there are $2^{k}$ possible results of step (ii). To complete the proof, notice that $\Pi=\beta \Pi \gamma \alpha^{c}$ if and only if $\Pi \alpha^{-d}=\beta \Pi \alpha^{-d} \gamma$, where $c=2 d \bmod m$. The $m$ choices for $d$ (and hence for $c$ ) give the factor of $m$ in $m 2^{k} k$ !.

Corresponding to Corollary 4.3 we have:

COROLLARY 5.2. There are exactly $m 2^{k} k$ ! m-tone rows $\Pi$ for which $\square(\Pi)$ has fewer than $4 m$ distinct members, and in such cases the cardinality of $\square(\Pi)$ is $2 m$.

THeOREM 5.3. The set-complex $\square\left(\mathscr{P}_{m}\right)$ has cardinality

$$
\left(m !+m 2^{k} k !\right) 4 m=(m-1) ! / 4+2^{k-2} k !
$$

Proof. Use Burnside's Lemma as in the proof of Theorem 4.4.

TheOREM 5.4. The cardinality of $\left\{\square(\Pi): \Pi \in \mathscr{P}_{m}\right.$ and $\left.|\square(\Pi)|<4 m\right\}=\{\square(\Pi)$ : $\Pi \in \mathscr{P}_{m}$ and $\left.|\square(\Pi)|=2 m\right\}$ is $2^{k-1} k$ !.

Proof. Similar to that of Theorem 4.5.

\section{Examples}

In this section we illustrate the results of the theorems in Sections 4 and 5 .

Example 1 . There are exactly 6 three-tone rows $\Pi$ for which $\Pi=\beta \Pi \alpha^{-2 \pi_{0}} \gamma \alpha^{a}$ for some $a$. They are (using the method of proof of Theorem 5.1):

$$
(1,0,2), \quad(2,0,1), \quad(2,1,0), \quad(0,1,2), \quad(0,2,1), \quad(1,2,0) \text {. }
$$

These are all the three-tone rows. For all 6 three-tone rows it is true (compare with Corollary 5.2) that $\square(\Pi)$ has 6 , not 12, distinct members. According to Theorem 5.3, there is $(3-1) ! / 4+2^{1-2} 1 !=1$ set-complex in $\square\left(\mathscr{P}_{3}\right)$, and Theorem 
5.4 again assures us that it has only 6 members. Of course, the only set-complex is the above set of 6 three-tone rows. It may be written in the following form:

\begin{tabular}{lll}
\hline 0 & 1 & 2 \\
\hline 1 & 0 & 2 \\
2 & 1 & 0 \\
0 & 2 & 1 \\
\hline
\end{tabular}

Example 2. There are exactly 8 four-tone rows $\Pi$ for which $\Pi=\beta \Pi \alpha^{a}$, and in all these cases $a=2$. They are (using the method of proof of Theorem 4.1):

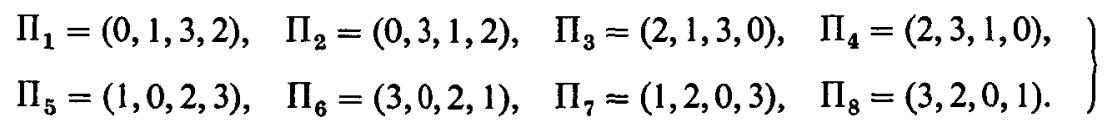

There are exactly 16 four-tone rows $\Pi$ for which $\Pi=\beta \Pi \alpha^{-2 \pi_{0}} \gamma \alpha^{a}$ for some $a$. They are (using the method of proof of Theorem 4.2):

$$
\begin{array}{clll}
\Pi_{9}=(0,2,3,1), & \Pi_{10}=(0,3,2,1), & \Pi_{11}=(1,2,3,0), & \Pi_{12}=(1,3,2,0), \\
\Pi_{13}=(2,0,1,3), & \Pi_{14}=(3,0,1,2), & \Pi_{15}=(2,1,0,3), & \Pi_{16}=(3,1,0,2), \\
\Pi_{17}=(1,3,0,2), & \Pi_{18}=(1,0,3,2), & \Pi_{19}=(2,3,0,1), & \Pi_{20}=(2,0,3,1), \\
\Pi_{21}=(3,1,2,0), & \Pi_{22}=(0,1,2,3), & \Pi_{23}=(3,2,1,0), & \Pi_{24}=(0,2,1,3) .
\end{array}
$$

Notice that the set (1) of four-tone rows is disjoint from the set (2). For all 24 four-tone rows $\Pi$, it is true (compare with Corollary 4.3 ) that $\square(\Pi)$ has 8 , not 16, distinct members. According to Theorem 4.4 , there are $\left(4 !+2^{2}(2+1) !\right) /(4 \cdot 4)=3$ distinct set-complexes in $\square\left(\mathscr{P}_{4}\right)$, and Theorem 4.5 again assures us that each of

\begin{tabular}{|c|c|c|c|c|c|c|c|c|c|c|c|}
\hline 0 & 1 & 2 & 3 & 0 & 1 & 2 & 3 & 0 & 1 & 2 & 3 \\
\hline 0 & 1 & 3 & 2 & 0 & 2 & 3 & 1 & 0 & 3 & 2 & 1 \\
\hline 3 & 0 & 2 & 1 & 2 & 0 & 1 & 3 & 1 & 0 & 3 & 2 \\
\hline 1 & 2 & 0 & 3 & 1 & 3 & 0 & 2 & 2 & 1 & 0 & 3 \\
\hline 2 & 3 & 1 & 0 & 3 & 1 & 2 & 0 & 3 & 2 & 1 & 0 \\
\hline
\end{tabular}
the three has 8 , rather than 16 , distinct members. The three set-complexes are those sequences in (1) plus

$\left\{\Pi_{9}, \Pi_{17}, \Pi_{13}, \Pi_{21}, \Pi_{24}, \Pi_{12}, \Pi_{20}, \Pi_{16}\right\} \quad$ and $\quad\left\{\Pi_{10}, \Pi_{18}, \Pi_{15}, \Pi_{23}, \Pi_{22}, \Pi_{11}, \Pi_{19}, \Pi_{14}\right\}$.

These three set-complexes can be written in the following form: 
Example 3. There are exactly $12 !=479,001,600$ twelve-tone rows. Of these (1) $2^{6} \cdot 6 !=46,080$ are transpositions of their own retrogrades (in each case, transposition by 6 ); (2) $6 \cdot 2^{6} \cdot 6 !=276,480$ are transpositions of their own retrograde-inversions (for each $c=1,3,5,7,9$, and 11 , there are 46,080 twelvetone rows which are transpositions of their own retrograde-inversion by $c$ ). According to Corollary 4.3 there are, therefore, 322,560 twelve-tone rows $\Pi$ for which $\square(\Pi)$ has 24 , rather than 48 , distinct members. The number of distinct $\square(\Pi)$ is, by Theorem 4.4 , equal to $\left(12 !+2^{6} \cdot(6+1) !\right) /(4 \cdot 12)=9,985,920$. By Theorem $4.5,2^{6-1} \cdot(6+1) ! / 12=13,440$ of these have 24 , rather than 48 , distinct members. Note that

$$
(13,440 \times 24)+(9,972,480 \times 48)=479,001,600 .
$$

Table 2 sets out, for each $m$ between 3 and 15, the appropriate number of $m$ ! $m$-tone rows which are transpositions either of their own retrogrades or of their own retrograde-inversions. Also included in Table 2 is the number of distinct set-complexes, divided according to whether the set-complex contains $2 m$ or $4 m$ distinct $m$-tone rows. It is worth noting the following identity:

$$
m !=((\sharp \square(\Pi):|\square(\Pi)|=2 m\}) \times(2 m))+((\sharp \square(\Pi):|\square(\Pi)|=4 m\}) \times(4 m)),
$$

a special case of which is illustrated at the end of Example 3.

\section{Results for large $m$}

For $m>15$, the values that would appear in Table 2 are too large to print exactly or even to comprehend easily. However, using Stirling's formula for $m$ ! we can make several simple approximations for the entries with large values of $m$. The most interesting results are the following. The proofs are omitted.

(1) The square of the number of $m$-tone rows which are transpositions of their own retrogrades behaves asymptotically like the total number of $m$-tone rows times $(\pi m / 2)^{2}$ for $m$ even and is exactly 0 for $m$ odd.

(2) The square of the number of $m$-tone rows which are transpositions of their own retrograde-inversions behaves asymptotically like the total number of $m$-tone rows times $\left(\pi m^{3} / 2\right)^{\frac{1}{1}} f_{m}$, where $f_{m}$ is $m / 4$ if $m$ is even and is 1 if $m$ is odd.

(3) The number of distinct set-complexes behaves asymptotically like $(m-1) ! / 4$ for all $m$.

(4) The square of the number of set-complexes with $2 m$ distinct $m$-tone rows behaves asymptotically like the number of distinct set-complexes times $(\pi m / 2)^{\frac{l}{2}} f_{m}$, where $f_{m}$ is defined in (2). 


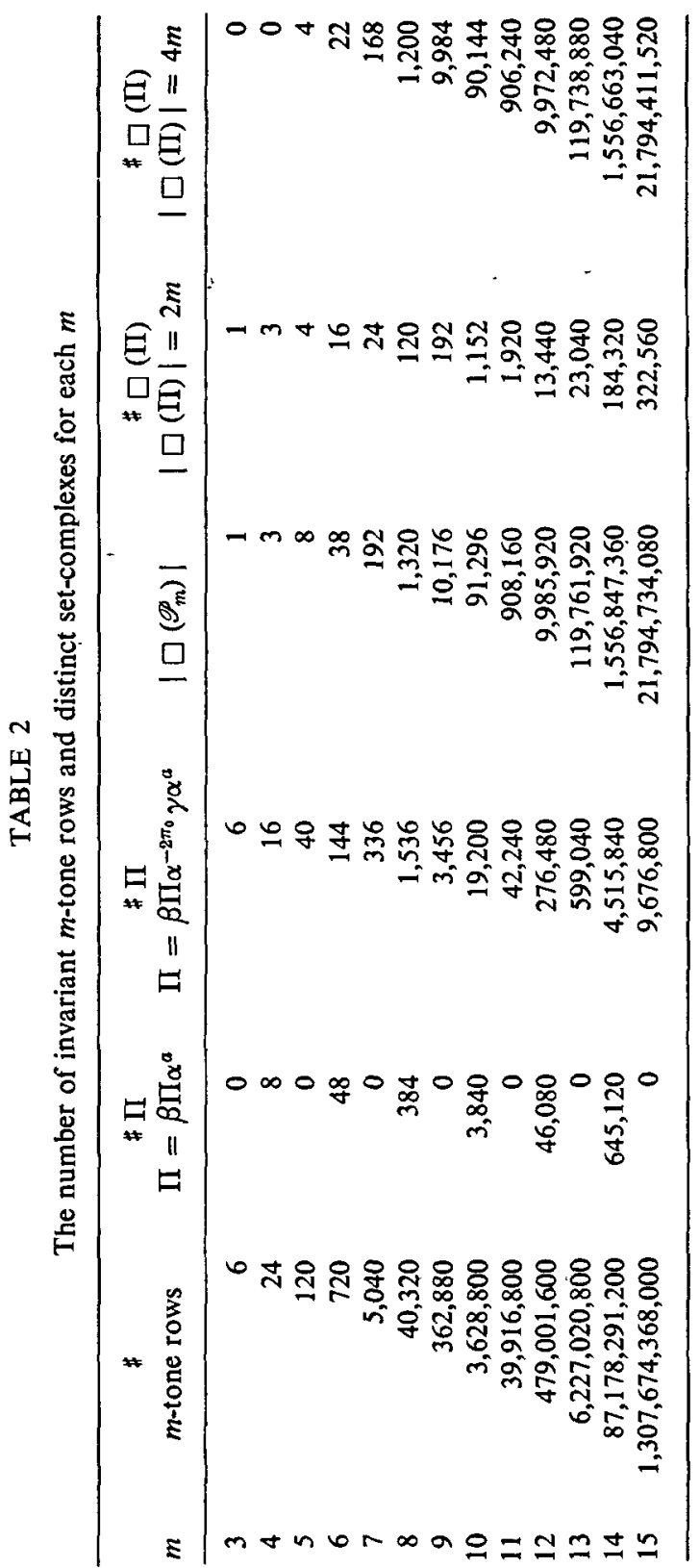




\section{Additional remarks}

It is important to note that the results of this paper apply not only to $m$-tone rows constructed from a single $m$-tonal scale (that is, from a single octave), but also to compositions in which each note of an $m$-tone row can be stated in any octave that the composer desires; indeed, this is part of the Schoenberg system. The extension is straightforward: the composer states the number of octaves that he is willing to consider in his choice of notes, say five; since there are $m$ ! distinct $m$-tone rows that can be obtained from a single octave, there must be $5^{m} m$ ! $m$-tone rows that can be obtained from five octaves. Similar modifications apply to each of the results of Sections 4 and 5 .

In a further paper (see [7]) the authors present an efficient method for generating all twelve-tone rows which are transpositions of their own retrograde-inversions. This is an alternative procedure to that outlined in the proof of Theorem 4.2. The method also applies to deriving all tone rows of even length which are transpositions of their own retrograde-inversions.

A related subject to that presented here concerns the medieval art of changeringing on church bells. We refer the interested reader to reference [8].

\section{Acknowledgements}

We should like to thank Professor Easley Blackwood of the Department of Music at the University of Chicago for introducing us to this subject and for his constant encouragement. Comments by Professor Irving Kaplansky on a previous draft of this paper proved invaluable, and discussions with Allen Moy, Ronald Thisted and Sandy Zabell were very helpful. We should also like to thank the Arnold Schoenberg Institute for providing us with reference [10].

Work on this paper was carried out while Alan J. Izenman was a Visiting Assistant Professor in the Department of Statistics, University of Chicago, Chicago, Illinois. Support for this research was provided in part by National Science Foundation Grants No. SOC72-05228 A04 and No. MCS72-04364 A04. James Fill is a National Science Foundation Fellow and Robert R. McCormick Fellow.

\section{References}

[1] M. Babbitt, "The function of set structure in the twelve-tone system", Unpublished manuscript, Department of Music, Princeton University (1946).

[2] M. Babbitt, "Some aspects of twelve-tone composition", The Score and I.M.A. Magazine 12 (1955), 53-61. 
[3] M. Babbitt, "Twelve-tone invariants as compositional determinants", The Musical Quarterly 46 (1960), 246-259.

[4] M. Babbitt, "Set structure as a compositional determinant", Journal of Music Theory 5 (1961), 72-92.

[5] M. Babbitt, "Since Schoenberg", Perspectives of New Music (Fall-Winter 1973, SpringSummer 1974, Special Double Issue), 3-28.

[6] J. Denes and A. D. Keedwell, Latin squares and their application (Academic Press, New York, 1974).

[7] J. A. Fill and A. J. Izenman, "The structure of RL-invariant twelve-tone rows", J. Austral. Math. Soc. Ser. B (to appear).

[8] T. L. Fletcher, "Campanological groups", American Mathematical Monthly (1956), 619-626.

[9] C. L. Liu, Introduction to combinatorial mathematics (McGraw-Hill, New York, 1968).

[10] J. Maegaard, "Schönbergs Zwölftonreihen", Die Musikforschung 29 (1976), 385-425.

[11] G. Perle, Serial composition and atonality (University of California Press, 2nd edition, 1968).

[12] A. Schoenberg, Style and idea (Philosophical Library, New York, 1950).

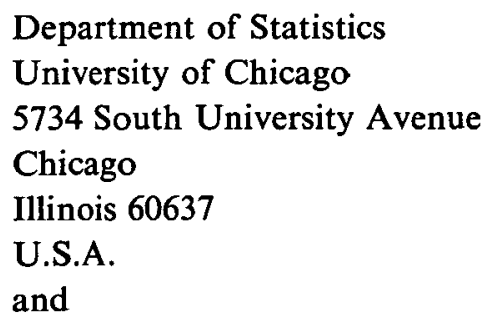

Department of Applied Statistics

School of Statistics

University of Minnesota

Saint Paul

Minnesota 55108

U.S.A. 